\title{
Mathematical modeling of tumor surface growth with necrotic kernels
}

\author{
Hua Zhang ${ }^{1}$, Jianjun $\mathrm{Tian}^{2}$, Ben $\mathrm{Niu}^{3}$, and Yuxiao Guo ${ }^{3}$ \\ ${ }^{1}$ Harbin Institute of Technology \\ ${ }^{2}$ New Mexico State University \\ ${ }^{3}$ Harbin Institute of Technology - Weihai
}

July 11, 2020

\begin{abstract}
A two-dimensional tumor-immune model with the time delay of the adaptive immune response is considered in this paper. The model accounts for the interaction between cytotoxic T lymphocytes (CTLs) and cancer cells on the surface of a solid tumor with a necrotic kernel. The system has three equilibria. Both zero and maximum tumor volume equilibria are unstable, while the behavior of the positive equilibriums is closely related to the ratio of the immune killing rate to tumor volume growth rate. The positive equilibrium is more likely to be locally asymptotically stable when the ratio is smaller than a critical value, and unstable otherwise. We also derive conditions to guarantee the existence of Hopf bifurcation at the positive equilibrium. Applying the center manifold reduction and normal form method, we obtain explicit formulas to determine the properties of Hopf bifurcations. The global continuation of local Hopf bifurcation is studied based on the coincidence degree theory. The results reveal that long immune delay can lead to oscillation dynamics. We also carry out detailed numerical analysis for parameters to illustrate our qualitative analysis. Numerically, we find that a shorter immune response time leads to a longer patient survival time and the period and amplitude of a stable periodic solution increase with the immune response time. When CTLs recruitment rate and death rate vary, we observe how the ratio metioned above and the first bifurcation value change numerically, which yields further insights to the tumor-immune dynamics.
\end{abstract}

\section{Hosted file}

template_MMAS.pdf available at https://authorea.com/users/341921/articles/468802-

mathematical-modeling-of-tumor-surface-growth-with-necrotic-kernels 
figures/kenel/kenel-eps-converted-to.pdf 
figures/k-r/k-r-eps-converted-to.pdf 
figures/d-kr/d-kr-eps-converted-to.pdf 
figures/rho-kr/rho-kr-eps-converted-to.pdf 
figures/survival/survival-eps-converted-to.pdf 
figures/stable/stable-eps-converted-to.pdf 
figures/stable-pha/stable-pha-eps-converted-to.pdf 
figures/period/period-eps-converted-to.pdf 
figures/period-pha/period-pha-eps-converted-to.pdf 
figures/bif4(x)/bif4(x)-eps-converted-to.pdf 
figures/bif4(CTL)/bif4 (CTL)-eps-converted-to.pdf 
figures/d-tau/d-tau-eps-converted-to.pdf 
figures/rho-tau/rho-tau-eps-converted-to.pdf 
figures/r-tau/r-tau-eps-converted-to.pdf 
figures $/ k-t a u / k-t a u-e p s-c o n v e r t e d-t o . p d f$ 
figures/k-r-tau (2)/k-r-tau (2)-eps-converted-to.pdf 